Is weak pseudo-Hermiticity weaker than pseudo-Hermiticity?

Ali Mostafazadeh

Citation: Journal of Mathematical Physics 47, 092101 (2006); doi: 10.1063/1.2338144

View online: http://dx.doi.org/10.1063/1.2338144

View Table of Contents: http://aip.scitation.org/toc/jmp/47/9

Published by the American Institute of Physics

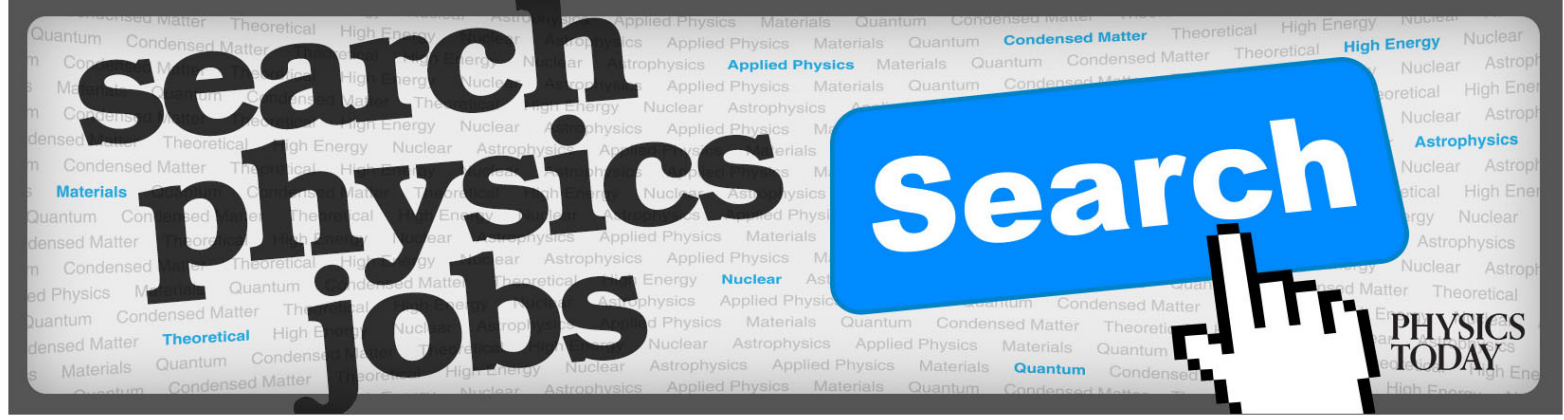




\title{
Is weak pseudo-Hermiticity weaker than pseudo-Hermiticity?
}

\author{
Ali Mostafazadeh ${ }^{\text {a) }}$ \\ Department of Mathematics, Koç University, 34450 Sariyer, Istanbul, Turkey
}

(Received 2 May 2006; accepted 18 July 2006; published online 5 September 2006)

For a weakly pseudo-Hermitian linear operator, we give a spectral condition that ensures its pseudo-Hermiticity. This condition is always satisfied whenever the operator acts in a finite-dimensional Hilbert space. Hence weak pseudo-Hermiticity and pseudo-Hermiticity are equivalent in finite-dimensions. This equivalence extends to a much larger class of operators. Quantum systems whose Hamiltonian is selected from among these operators correspond to pseudo-Hermitian quantum systems possessing certain symmetries. (C) 2006 American Institute of Physics.

[DOI: $10.1063 / 1.2338144]$

\section{INTRODUCTION}

References 1 and 2 discuss a notion of a pseudo-Hermitian operator that has proven to be a convenient tool in the study of $\mathcal{P} \mathcal{T}$-symmetric Hamiltonians. ${ }^{3-7}$ It also plays a central role in solving some of the basic problems of relativistic quantum mechanics and quantum cosmology ${ }^{8}$ and revealing some interesting analogies between quantum mechanics and general relativity. ${ }^{9}$ The following is a mathematically precise description of this notion. ${ }^{1}$

Definition 1: A densely defined linear operator $H: \mathcal{H} \rightarrow \mathcal{H}$ acting in a separable Hilbert space $\mathcal{H}$ is said to be pseudo-Hermitian if there exists a Hermitian automorphism $\eta: \mathcal{H} \rightarrow \mathcal{H}$ satisfying

$$
H^{\dagger}=\eta H \eta^{-1},
$$

where $H^{\dagger}$ denotes the adjoint of $H$. (Throughout this paper, "Hermitian" means "self-adjoint," e.g., $\eta^{\dagger}=\eta$.)

For a discussion of the earlier uses of the term pseudo-Hermitian in the context of indefinitemetric theories see Ref. 10.

Note that an automorphism is by definition an everywhere-defined, one-to-one, and onto linear operator. Moreover, an everywhere-defined Hermitian linear operator is necessarily bounded (this is known as the Hellinger-Toeplitz theorem ${ }^{11}$ ), and a bounded one-to-one onto linear map has a bounded inverse (this is known as the inverse mapping theorem ${ }^{11}$ or Banach's theorem ${ }^{12}$ ). As a result, if one adopts the definition of an invertible operator that identifies the latter with a one-toone, onto linear map with a bounded inverse, ${ }^{13-15}$ then a linear operator is everywhere-defined, Hermitian, and invertible if and only if it is a Hermitian automorphism. Usually in physics literature one ignores the technical issues associated with the domain of the operators and uses "Hermitian automorphism" and "Hermitian invertible linear map" synonymously. Another more familiar term used for such an operator particularly in the context of pseudo-Hermitian operators is "pseudo-metric."

The operator equation (1) in particular implies that the domain of its both sides must coincide. In light of the fact that $\eta$ is everywhere-defined, this means

\footnotetext{
${ }^{a)}$ Electronic mail: amostafazadeh@ku.edu.tr
} 


$$
\eta(\mathcal{D}(H))=\mathcal{D}\left(H^{\dagger}\right),
$$

where $\mathcal{D}(L)$ denotes the domain of a linear operator $L: \mathcal{H} \rightarrow \mathcal{H}$, and $L(\mathcal{S})$ stands for the image of a subset $\mathcal{S} \subseteq \mathcal{H}$ under $L$.

Definition 1 is a direct generalization of the notion of a self-adjoint operator, for the latter corresponds to a pseudo-Hermitian operator admitting the identity operator $I: \mathcal{H} \rightarrow \mathcal{H}$ as a pseudometric. (We may similarly generalize the notion of a symmetric operator ${ }^{11}$ by replacing (2) with $\eta(\mathcal{D}(H)) \subseteq \mathcal{D}\left(H^{\dagger}\right)$ and requiring that (1) holds in $\eta(\mathcal{D}(H))$.)

In Ref. 4, Solombrino has slightly weakened the defining condition of a pseudo-Hermitian operator by relaxing the requirement of the Hermiticity of $\eta$. This leads to the following notion of weak pseudo-Hermiticity.

Definition 2: A linear operator $H: \mathcal{H} \rightarrow \mathcal{H}$ acting in a separable Hilbert space $\mathcal{H}$ is said to be weakly pseudo-Hermitian if there exists an everywhere-defined, bounded, invertible, linear map (i.e., a bounded automorphism) $\eta_{w}: \mathcal{H} \rightarrow \mathcal{H}$ satisfying

$$
H^{\dagger}=\eta_{w} H \eta_{w}^{-1} .
$$

Again (3) implies

$$
\eta_{w}(\mathcal{D}(H))=\mathcal{D}\left(H^{\dagger}\right) .
$$

The basic motivation for introducing weak pseudo-Hermiticity is that the Hermiticity of $\eta$ in (1) does not play any significant role in establishing the spectral characterization theorem(s) for diagonalizable pseudo-Hermitian operators with a discrete spectrum. ${ }^{1,2,4}$ This suggests, at least for diagonalizable operators with a discrete spectrum, that pseudo-Hermiticity and weak pseudoHermiticity are equivalent conditions. ${ }^{4}$ In Ref. 5 Bagchi and Quesne explore the relationship between these two concepts and use the term "complementary" to describe it. Though it is not made explicit in their analysis, their approach can be consistently applied only to a restricted class of bounded automorphisms $\eta$, namely to those for which $\eta+\eta^{\dagger}$ is also an automorphism. More recently, Znojil ${ }^{7}$ has suggested that considering weak-pseudo-Hermitian Hamiltonians may provide further insight into the current search for potential applications of non-Hermitian Hamiltonians in quantum mechanics.

The purpose of this paper is to conduct a careful reexamination of the relationship between weak pseudo-Hermiticity and pseudo-Hermiticity for a general not necessarily diagonalizable linear operator. We will establish the equivalence of these concepts for a large class of linear operators including all linear operators that act in a finite-dimensional Hilbert space, i.e., matrix Hamiltonians.

Before starting our analysis we introduce our conventions and notation.

- $\mathcal{H}$ denotes a separable Hilbert space.

- For any linear operator $H: \mathcal{H} \rightarrow \mathcal{H}, \mathfrak{U}_{H}$ stands for the set of all bounded automorphisms $\eta_{w}: \mathcal{H} \rightarrow \mathcal{H}$ satisfying (3). Therefore, $H$ is weakly pseudo-Hermitian if $\mathfrak{U}_{H} \neq \varnothing$. It is pseudoHermitian if $\mathfrak{U}_{H}$ contains a Hermitian element.

- For any bounded operator $B: \mathcal{H} \rightarrow \mathcal{H},\|B\|$ denotes the norm of $B$.

\section{A CAREFUL LOOK AT WEAK PSEUDO-HERMITICITY}

First we present some useful facts.

Proposition 1: Let $H: \mathcal{H} \rightarrow \mathcal{H}$ be a weakly pseudo-Hermitian linear operator. Then for all $\eta_{w}$, $\eta_{w}^{\prime} \in \mathfrak{U}_{H}, \eta_{w}^{-1} \eta_{w}^{\prime}$ is a bounded automorphism commuting with $H$.

Proof: Let $\eta_{w}, \eta_{w}^{\prime} \in \mathfrak{U}_{H}$. Because both $\eta_{w}$ and $\eta_{w}^{\prime}$ are bounded, one-to-one, and onto, so are $\eta_{w}^{-1}$ and $\eta_{w}^{-1} \eta_{w}^{\prime}$. Furthermore, as shown in Ref. 1, in view of (3) and its analog satisfied by $\eta_{w}^{\prime}$, we have $\left[\eta_{w}^{-1} \eta_{w}^{\prime}, H\right]=0$.

Proposition 2: Let $H: \mathcal{H} \rightarrow \mathcal{H}$ be a closed weakly pseudo-Hermitian linear operator and $\eta_{w}$ $\in \mathfrak{U}_{H}$. Then $\eta_{w}^{\dagger} \in \mathfrak{U}_{H}$ provided that $\eta_{w}^{\dagger} \mathcal{D}(H)=\mathcal{D}\left(H^{\dagger}\right)$. In this case 


$$
A:=\eta_{w}^{-1} \eta_{w}^{\dagger}
$$

is a bounded automorphisms commuting with $H$. (Although Definitions 1 and 2 do not require $H$ to be a closed operator, this requirement is necessary to derive many of the useful properties of pseudo-Hermitian and weakly pseudo-symmetric operators. Here we need it to assure that $H^{\dagger \dagger}$ $=H .{ }^{16}$ )

Proof: Let $\eta_{w} \in \mathfrak{U}_{H}$ be such that $\eta_{w}^{\dagger} \mathcal{D}(H)=\mathcal{D}\left(H^{\dagger}\right) . \eta_{w}$ satisfies (3) or equivalently

$$
\eta_{w} H=H^{\dagger} \eta_{w} .
$$

This in particular implies

$$
\eta_{w}^{-1} \mathcal{D}\left(H^{\dagger}\right)=\mathcal{D}\left(H^{\dagger} \eta_{w}\right)=\mathcal{D}\left(\eta_{w} H\right)=\mathcal{D}(H) .
$$

Now, take the adjoint of both sides of this equation. [Note that for a pair of (densely defined) linear operators $A, B: \mathcal{H} \rightarrow \mathcal{H}$ that are not bounded and everywhere-defined, the relation $(A B)^{\dagger}=B^{\dagger} A^{\dagger}$ does not hold in general (Ref. 14, Sec. 7.7).] Because $\mathcal{D}\left(\eta_{w} H\right)=\mathcal{D}(H)$ is dense and $\eta_{w}$ is bounded and everywhere-defined, we have (Ref. 14, Sec. 7.7)

$$
\left(\eta_{w} H\right)^{\dagger}=H^{\dagger} \eta_{w}^{\dagger},
$$

or alternatively

$$
H^{\dagger}=\left(\eta_{w} H\right)^{\dagger} \eta_{w}^{\dagger-1} .
$$

Furthermore, as explained in Ref. 14, Sec. 7.7, because $\mathcal{D}\left(H^{\dagger} \eta_{w}\right)=\eta_{w}^{-1} \mathcal{D}\left(H^{\dagger}\right)=\mathcal{D}(H)$ is dense, $\mathcal{D}\left(\eta_{w}^{\dagger} H\right) \subseteq \mathcal{D}\left(\left(H^{\dagger} \eta_{w}\right)^{\dagger}\right)$ and

$$
\left(H^{\dagger} \eta_{w}\right)^{\dagger} \psi=\eta_{w}^{\dagger} H \psi \quad \text { for all } \psi \in \mathcal{D}\left(\eta_{w}^{\dagger} H\right)=\mathcal{D}(H) .
$$

This in turn means that

$$
\left(H^{\dagger} \eta_{w}\right)^{\dagger} \eta_{w}^{\dagger-1} \phi=\eta_{w}^{\dagger} H \eta_{w}^{\dagger-1} \phi \quad \text { for all } \phi \in \eta_{w}^{\dagger} \mathcal{D}(H) .
$$

Therefore, in view of the hypothesis: $\eta_{w}^{\dagger} \mathcal{D}(H)=\mathcal{D}\left(H^{\dagger}\right)$ and Eqs. (6), (9), and (10) (as envisaged in Ref. 5)

$$
H^{\dagger}=\eta_{w}^{\dagger} H \eta_{w}^{\dagger-1} .
$$

This together with the fact that the adjoint $\left(\eta_{w}^{\dagger}\right)$ of a bounded automorphism $\left(\eta_{w}\right)$ is a bounded automorphism establish $\eta_{w}^{\dagger} \in \mathfrak{U}_{H}$. The fact that $[A, H]=0$ follows from Proposition 1.

Proposition 3: Let $H$ and $A$ be as in Proposition 2 and $r_{A}:=\lim _{n \rightarrow \infty}\left\|A^{n}\right\|^{1 / n}$ be the spectral radius ${ }^{17}$ of $A$. Then the spectrum $\sigma_{A}$ of $A$ lies in the annulus centered at $0 \in \mathrm{C}$ and having as its inner and outer radii $r_{A}^{-1}$ and $r_{A}$, respectively, i.e.,

$$
\sigma_{A} \subseteq\left\{z \in \mathbb{C}\left|r_{A}^{-1} \leqslant\right| z \mid \leqslant r_{A}\right\} .
$$

In particular, $\|A\| \geqslant r_{A} \geqslant 1$.

Proof: According to Proposition 2, $A$ is a bounded invertible linear map. This implies that $A^{\dagger}$ and $A^{-1}$ are bounded operators, and the following identities are satisfied: $:^{17,14}$

$$
\sigma_{A^{\dagger}}=\left\{\lambda \in \mathbb{C} \mid \lambda^{*} \in \sigma_{A}\right\}, \quad \sigma_{A^{-1}}=\left\{\lambda \in \mathbb{C} \mid \lambda^{-1} \in \sigma_{A}\right\} .
$$

Furthermore, because $A^{-1}=\eta_{w}^{\dagger-1} \eta_{w}, A^{\dagger}=\eta_{w} \eta_{w}^{-1 \dagger}=\eta_{w} A^{-1} \eta_{w}^{-1}$, and $\eta_{w}$ is invertible, we have $\sigma_{A^{\dagger}}$ $=\sigma_{A^{-1}}{ }^{15}$ Combining this result with (14), we find that for all $\lambda \in \sigma_{A}, 1 / \lambda^{*} \in \sigma_{A}$. Next, we recall that for all $\mu \in \sigma_{A},|\mu| \leqslant r_{A}$. Applying this inequality for $\mu=\lambda$ and $1 / \lambda^{*}$, we then find $r_{A}^{-1} \leqslant|\lambda|$ $\leqslant r_{A}$ for all $\lambda \in \sigma_{A}$. (Because there is always $\Lambda \in \sigma_{A}$ such that $|\Lambda|=r_{A}$ and $1 / \Lambda^{*} \in \sigma_{A}, \sigma_{A}$ intersects both the circles $|z|=r_{A}$ and $|z|=r_{A}^{-1}$.) This establishes (13). Finally, because the spectrum of every bounded operator is nonempty, we must have $r_{A}^{-1} \leqslant r_{A}$, which in turn implies $r_{A} \geqslant 1$. The fact that 
$r_{A} \leqslant\|A\|$ is well-known. ${ }^{17}$

The following is our main result. It links the equivalence of weak pseudo-Hermiticity and pseudo-Hermiticity of a large class of linear operators $H$ with the existence of an $\eta_{w} \in \mathfrak{U}_{H}$ such that the unit circle $S^{1}:=\left\{e^{i \varphi} \in \mathrm{C} \mid \varphi \in[0,2 \pi)\right\}$ is not a subset of $\sigma_{A}$. Note that Proposition 3 does not rule out this possibility.

Theorem 1: Let $H: \mathcal{H} \rightarrow \mathcal{H}$ be a closed weakly pseudo-Hermitian linear operator acting in a separable Hilbert space $\mathcal{H}$. Then $H$ is pseudo-Hermitian, if there is $\eta_{w} \in \mathfrak{U}_{H}$ such that $\eta_{w}^{\dagger} \mathcal{D}(H)$ $=\mathcal{D}\left(H^{\dagger}\right)$ and the unit circle $S^{1}$ is not a subset of the spectrum $\sigma_{A}$ of $A:=\eta_{w}^{-1} \eta_{w}^{\dagger}$.

Proof: Let $\eta_{w} \in \mathfrak{U}_{H}$ be such that $\eta_{w}^{\dagger} \mathcal{D}(H)=\mathcal{D}\left(H^{\dagger}\right)$, and $\vartheta \in[0,2 \pi)$ be arbitrary. Then according to Proposition 2, $\eta_{w}^{\dagger} \in \mathfrak{U}_{H}$, and both (3) and (12) hold. Expressing these equations in the form

$$
\begin{aligned}
& \eta_{w} H=H^{\dagger} \eta_{w}, \\
& \eta_{w}^{\dagger} H=H^{\dagger} \eta_{w}^{\dagger},
\end{aligned}
$$

multiplying both sides of (15) and (16), respectively, by $i e^{i \vartheta}$ and $-i e^{-i \vartheta}$, and adding the resulting equations side by side, we find

$$
\eta(\vartheta) H=H^{\dagger} \eta(\vartheta)
$$

where

$$
\eta(\vartheta):=i\left(e^{i \vartheta} \eta_{w}-e^{-i \vartheta} \eta_{w}^{\dagger}\right) \quad \text { for all } \vartheta \in[0,2 \pi) .
$$

The operator $\eta(\vartheta)$ is manifestly Hermitian. It is also everywhere-defined and bounded, because both $\eta_{w}$ and $\eta_{w}^{\dagger}$ share these properties. But it need not be invertible. We can express $\eta(\vartheta)$ in the form

$$
\eta(\vartheta)=-i e^{-i \vartheta} \eta_{w}\left(A-e^{2 i \vartheta} I\right),
$$

where $I$ stands for the identity operator acting on $\mathcal{H}$. Clearly because $\eta_{w}$ is invertible, $\eta(\vartheta)$ is invertible if and only if $A-e^{2 i \vartheta} I$ is invertible. By the definition of the spectrum of a linear operator, ${ }^{11-17}$ the latter condition is equivalent to $e^{2 i \vartheta} \notin \sigma_{A}$. If $S^{1} \subseteq \sigma_{A}$, there is $\vartheta_{\star} \in[0, \pi)$ such that $e^{2 i \vartheta_{\star}} \notin \sigma_{A}$. Therefore, $\eta_{\star}:=\eta\left(\vartheta_{\star}\right)$ is invertible; $\mathfrak{U}_{H}$ includes a Hermitian operator $\eta_{\star}$; and $H$ is pseudo-Hermitian.

Corollary: A linear operator acting in a finite-dimensional Hilbert space is weakly pseudoHermitian if and only if it is pseudo-Hermitian. (As pointed out by the referee, this is a known result. ${ }^{18}$ )

Proof: According to Definitions 1 and 2, every pseudo-Hermitian operator is weakly pseudoHermitian. The converse holds for an operator acting in a finite-dimensional Hilbert space, because in this case all the operators are everywhere-defined (bounded and hence closed) and $\sigma_{A}$ of Theorem 1 is a finite set. Hence, it cannot include $S^{1}$ as a subset.

In summary, a weakly pseudo-Hermitian linear operator may fail to be pseudo-Hermitian, if it acts in an infinite-dimensional space and for every $\eta_{w} \in \mathfrak{U}_{H}$ either $\eta_{w}^{\dagger} \mathcal{D}(H) \neq \mathcal{D}\left(H^{\dagger}\right)$ or $S^{1} \subseteq \sigma_{A}$ where $A:=\eta_{w}^{-1} \eta_{w}^{\dagger}$. The latter condition seems to be very difficult to satisfy.

\section{EXAMPLES}

Consider the following bounded automorphism that is employed in Ref. 7:

$$
\eta_{w}=\left(\begin{array}{lll}
0 & 0 & \mathcal{P} \\
\mathcal{P} & 0 & 0 \\
0 & \mathcal{P} & 0
\end{array}\right),
$$

where $\mathcal{P}$ is the usual parity operator acting in $L^{2}(\mathbb{R})$, the Hilbert space is $L^{2}(\mathbb{R}) \oplus L^{2}(\mathbb{R}) \oplus L^{2}(\mathbb{R})$, a three-component representation of the state vectors is used, and $H=\left(H_{i j}\right)$ is a $3 \times 3$ matrix of 
differential operators $H_{i j}$ such that $\mathcal{P D}(H)=\mathcal{D}(H)=\mathcal{D}\left(H^{\dagger}\right)$. It is not difficult to see that $\vartheta_{\star}$ $=3 \pi / 2$ fulfills the conditions of Theorem 1 , and

$$
\eta_{\star}:=\eta\left(\frac{3 \pi}{2}\right)=\eta_{w}+\eta_{w}^{\dagger}=\left(\begin{array}{ccc}
0 & \mathcal{P} & \mathcal{P} \\
\mathcal{P} & 0 & \mathcal{P} \\
\mathcal{P} & \mathcal{P} & 0
\end{array}\right)
$$

is a genuine pseudo-metric belonging to $\mathfrak{U}_{H}$. Indeed, it is not only everywhere-defined, bounded, Hermitian, and one-to-one, but it is also onto and its inverse is bounded. This can be directly checked. Alternatively, we may apply Theorem 1 and show that $S^{1} \nsubseteq \sigma_{A}$. It is very easy to compute the symmetry generator (5):

$$
A=\left(\begin{array}{lll}
0 & 0 & 1 \\
1 & 0 & 0 \\
0 & 1 & 0
\end{array}\right)
$$

where 1 is viewed as the identity operator acting in $L^{2}(\mathbb{R})$. Clearly, $\sigma_{A}=\left\{1, e^{2 i \pi / 3}, e^{4 i \pi / 3}\right\}$. Hence $S^{1} \nsubseteq \sigma_{A}$ and $\eta_{\star}$ is invertible.

This calculation shows that the systems considered in Ref. 7 can be identified with $\eta_{\star}$-pseudo-Hermitian Hamiltonians acting in $L^{2}(\mathbb{R}) \oplus L^{2}(\mathbb{R}) \oplus L^{2}(\mathbb{R})$ and commuting with $A$, where $\eta_{\star}$ and $A$ are, respectively, given by (21) and (22). These systems can be studied without any reference to weak pseudo-Hermiticity.

Another probably more interesting example is $\eta_{w}: C^{2} \rightarrow C^{2}$ that is defined by its standard matrix representation according to

$$
\eta_{w}=\left(\begin{array}{cc}
1 & 1 \\
-1 & i
\end{array}\right)
$$

The symmetry generator (5) and the most general Hamiltonian $H: C \rightarrow C$ satisfying (6) have the following standard matrix representations:

$$
\begin{gathered}
A=\left(\begin{array}{cc}
i & 0 \\
1-i & -1
\end{array}\right)=-i M_{1}-M_{2}, \\
H=\left(\begin{array}{cc}
a & 0 \\
i b & a+b
\end{array}\right)=a I+b M_{1},
\end{gathered}
$$

where

$$
M_{1}:=\left(\begin{array}{cc}
0 & 0 \\
i & 1
\end{array}\right), \quad M_{2}:=\left(\begin{array}{cc}
1 & 0 \\
-i & 0
\end{array}\right)
$$

$I$ is the identity matrix, and $a, b \in \mathrm{R}$ are arbitrary. Clearly, $A$ and $H$ commute for all $a, b \in \mathbb{R}$.

We can also easily compute $A-e^{2 i \vartheta} I$. It turns out to be noninvertible only for $\vartheta$ $=\pi / 4, \pi / 2,5 \pi / 4,3 \pi / 2$. This in turn means that $\eta(\vartheta)$ is noninvertible for these four values of $\vartheta$. In particular, $\eta(3 \pi / 2)=\eta_{w}+\eta_{w}^{\dagger}$ that is considered in Ref. 5 is not invertible. (The possibility that given an invertible operator $\eta_{w}$ the operators $\eta_{w} \pm \eta_{w}^{\dagger}$ may fail to be invertible seems to be overlooked in Ref. 5.)

In general, $\eta(\vartheta)$ has the following explicit form: 


$$
\eta(\vartheta)=2 c\left(\begin{array}{cc}
-t & i \\
-i & -1
\end{array}\right)
$$

where $c:=\cos \vartheta$ and $t:=\tan \vartheta$. In terms of $c$ and $t$ the invertibility condition: $\vartheta \notin\{\pi / 4, \pi / 2,5 \pi / 4,3 \pi / 2\}$, takes the simple form: $c \neq 0$ and $t \neq 1$.

Having obtained an infinite class of pseudo-metric operators $\eta(\vartheta)$ that render the Hamiltonians of the form (25) pseudo-Hermitian, we can construct the following family of symmetry generators: $:^{1,6}$

$$
A\left(r, t_{1}, t_{2}\right):=\eta\left(\vartheta_{2}\right)^{-1} \eta\left(\vartheta_{1}\right)=r\left(\begin{array}{cc}
1-t_{1} & 0 \\
i\left(t_{1}-t_{2}\right) & 1-t_{2}
\end{array}\right)=r\left(I-t_{2} M_{1}-t_{1} M_{2}\right),
$$

where $r:=\cos \vartheta_{1} /\left(\cos \vartheta_{2}-\sin \vartheta_{2}\right) \neq 0$ and $t_{i}:=\tan \vartheta_{i} \neq 1$ for $i=1,2$. Comparing (25) and (27), we see that the only nontrivial symmetry generator for the system is $M_{2}$. We could reach the same conclusion using (24).

Finally, we note that $\eta(\alpha)$ is positive-definite whenever $c<0$ and $t>1$ which corresponds to $5 \pi / 4<\vartheta<3 \pi / 2$. In particular, $H$ is pseudo-Hermitian with respect to a set of positive-definite metric operators. According to Ref. 2, this implies that it is quasi-Hermitian ${ }^{19}$ and has real eigenvalues. The latter is easily seen from (25) where the eigenvalues appear as diagonal entries.

\section{CONCLUDING REMARKS}

In this paper, we have examined the relation between the notions of pseudo-Hermiticity and weak pseudo-Hermiticity. We have found a sufficient spectral condition that ensures whether a given weakly pseudo-Hermitian operator is pseudo-Hermitian. This condition which is not sensitive to the diagonalizability of the operator in question is trivially satisfied in finite-dimensional Hilbert spaces. Hence weak pseudo-Hermiticity and pseudo-Hermiticity are equivalent in finite dimensions. This equivalence extends to a large class of operators acting in infinite-dimensional Hilbert spaces. Our general results seem to indicate that further investigation of weak pseudoHermiticity is not likely to produce any substantial insight into the current study of the possible applications of non-Hermitian Hamiltonians in quantum mechanics.

\section{ACKNOWLEDGMENTS}

During the course of this work I have benefitted from helpful discussions with Varga Kalantarov. I would also like to thank the anonymous referee for bringing Ref. 18 to my attention and for correcting an error in a previous version of the paper.

\footnotetext{
${ }^{1}$ A. Mostafazadeh, J. Math. Phys. 43, 205 (2002).

${ }^{2}$ A. Mostafazadeh, J. Math. Phys. 43, 2814 (2002); 43, 3944 (2002).

${ }^{3}$ Z. Ahmed, Phys. Lett. A 290, 19 (2001); A. Mostafazadeh, J. Phys. A 36, 7081 (2003); quant-ph/0310164; A. Blasi, G. Scolarici, and L. Solombrino, 37, 4335 (2004); A. Mostafazadeh and A. Batal, J. Phys. A 37, 11645 (2004); S. Albeverio and S. Kuzhel, Lett. Math. Phys. 67, 223 (2004); A. Mostafazadeh, J. Phys. A 38, 3213 (2005); 38, 6557 (2005); 38, 8185 (2005); H. F. Jones, ibid. 38, 1741 (2005); H. F. Jones and J. Mateo, Phys. Rev. D 73, 085002 (2006); Y. Ben-Aryeh and R. Barak, Phys. Lett. A 351, 388 (2006); A. Mostafazadeh, J. Phys. A 39, 10171 (2006); D. Krejcirik, H. Bila, and M. Znojil, J. Phys. A 39, 10143 (2006).

${ }^{4}$ L. Solombrino, J. Math. Phys. 43, 5439 (2002).

${ }^{5}$ B. Bagchi and C. Quesne, Phys. Lett. A 301, 173 (2002).

${ }^{6}$ A. Mostafazadeh, J. Math. Phys. 44, 974 (2003).

${ }^{7}$ M. Znojil, Phys. Lett. A 353, 463 (2006).

${ }^{8}$ A. Mostafazadeh, Class. Quantum Grav. 20, 155 (2003); Ann. Phys. (N.Y.) 309, 1 (2004); A. Mostafazadeh and F. Zamani, Ann. Phys. (N.Y.) 321, 2183 (2006); 321, 2210 (2006).

${ }^{9}$ A. Mostafazadeh, Phys. Lett. A 320, 375 (2004); A. Mostafazadeh, J. Math. Phys. 47, 072103 (2006).

${ }^{10}$ A. Mostafazadeh, Czech. J. Phys. 53, 1079 (2003).

${ }^{11}$ M. Reed and B. Simon, Functional Analysis (Academic, San Diego, 1980), Vol. 1.

${ }^{12}$ A. N. Kolmogorov and S. V. Fomin, Introductory Real Analysis (Dover, New York, 1975).

${ }^{13}$ P. D. Hislop and I. M. Sigal, Introduction to Spectral Theory (Springer, New York, 1996).

${ }^{14}$ M. Schechter, Principles of Functional Analysis (American Mathematical Society, Providence, 2002).

${ }^{15}$ P. R. Halmos, A Hilbert Space Problem Book (Springer, New York, 1982).
} 
${ }^{16}$ K. Yosida, Functional Analysis (Springer, Berlin, 1995).

${ }^{17}$ T. Kato, Perturbation Theory for Linear Operators (Springer, Berlin, 1995).

${ }^{18}$ R. A. Horn and C. R. Johnson, Matrix Analysis (Cambridge University Press, Cambridge, 1999).

${ }^{19}$ F. G. Scholtz, H. B. Geyer, and F. J. W. Hahne, Ann. Phys. (N.Y.) 213, 74 (1992). 